\title{
The Phase Structure of the ISM in Galaxies
}

\author{
Mark G. Wolfire ${ }^{1}$ \\ ${ }^{1}$ Astronomy Department, University of Maryland, College Park, MD 20742, USA \\ email: mwolfire@astro.umd.edu
}

\begin{abstract}
Diffuse gas in the Galaxy is observed to exist as cold ( $T \sim 100 \mathrm{~K})$ neutral atomic gas $(\mathrm{CNM})$ and warm neutral atomic $(T \sim 8000 \mathrm{~K})$ gas (WNM). In addition to these "thermal" phases, gas can also exist as warm $(T \sim 8000 \mathrm{~K})$ ionized gas, cold $(T \sim 10 \mathrm{~K})$ molecular gas and in warm $(T \sim 100-500 \mathrm{~K})$ interface regions or Photodissociation Regions (PDRs) on the surfaces of molecular clouds. The same chemical and thermal processes that dominate in the PDRs associated with molecular clouds are also at work in the diffuse neutral gas. Two additional "phases" are gas associated with GMCs that has $\mathrm{H}_{2}$ but no or little CO, and short lived or transient phases such as shocks, shears, and turbulence. I will first review the different gas phases in the Galaxy, their physical conditions and their dominant cooling lines. I will also discuss the observations and theoretical modeling in support of turbulence versus thermal instability as the driving force in producing the "thermal" gas phase distributions. Rough estimates for the distribution of phases in the Galaxy and the origin of the dominant emission lines has been conducted by previous telescopes (e.g., COBE, BICE) but with low velocity and low spectral resolution. The distribution and mass of the various gas phases is important for sorting out the role of SN in setting ISM pressures and in driving ISM turbulence. In addition, understanding the Galactic phase distribution is important in interpreting observations of extragalactic systems in which beams encompass several emission components. I will review the potential for future observations by e.g., STO, SOFIA, and Herschel to detect and separate phases in Galactic and extragalactic systems.
\end{abstract}

Keywords. ISM: clouds, ISM: general, ISM: HII regions, galaxies: ISM

\section{Introduction}

What are some of the big ISM questions that might be answered by looking at phases? In this short written contribution I focus on the WNM and CNM phases. Which of the various ISM components dominates the [C II] emission and where? The warm $(T \sim 8000$ $\mathrm{K})$ neutral gas $(\mathrm{WNM})$ produces faint $[\mathrm{C} \mathrm{II}]$ and $[\mathrm{O} \mathrm{I}]$ and the cold $(T \sim 100 \mathrm{~K})$ neutral gas (CNM) produces mainly [C II]. The [C II] line mainly, but also with [O I, [N II], [C I], and $\mathrm{CO}$ are the dominant coolants of the ISM. Knowing the luminosity of the cooling lines, gives the energy input into the gas from either radiative heating or from turbulence or shocks. What is the ISM engine that drives gas into various phases? Part of answering this is to determine the distribution of CNM and WNM as well as the thermal pressure $P_{\text {th }}$. The distributions have implications for constraining the role of SN in setting pressures, the volume filling factor of hot gas, and shock processing of material.

\section{WNM and CNM Emissivity and Thermal Pressure}

We can estimate the emissivity in the WNM and CNM from phase diagrams ((Wolfire, et al. 2003)). In a plot of thermal pressure, $P_{\mathrm{th}} / k$ versus density $n$, and for an isobaric multiphase medium, the points of thermal stability correspond to the WNM and CNM phases. At $P_{\mathrm{th}}>P_{\max }$ gas is converted to the cold phase, but at $P_{\mathrm{th}}<P_{\mathrm{min}}$ the 
gas is converted to the warm phase. The cooling rate in the cold gas is a factor of 10 times larger than the cooling in the warm gas. So for comparable column densities, the [CII] emission from the cold gas is always brighter, and one can map out the CNM clouds in emission. Previously, CNM clouds were only seen in absorption using H I or UV absorption spectroscopy.

With this information one can 1)estimate the CNM contribution to the [C II] emission. 2) Along with HI get the WNM/CNM mass distribution as a function of position and test global models of the ISM. If SNRs dominate the topology of the ISM you expect more CNM than WNM. Most of the volume is filled with a hot ISM. On the other hand if SNRs are small and contained by magnetic fields then most of the volume is filled by WNM. 3)Along with H I the C II gives the thermal pressure in CNM clouds. Kulkarni \& Heiles (1987) suggested that this was a better way to get the thermal pressure than C I, or $\mathrm{C}$ II in absorption. This is because for a given $\mathrm{C}$ II cooling rate/H the pressure changes by only a factor of two for a wide range in temperatures.

Comparing thermal pressure in arm and interarm regions we can asses the role of thermal pressure in driving gas into cold phases which eventually leads to star formation. As expected for thermal instability (TI), does the the CNM/WNM rise as the thermal pressure goes up? If there is considerable WNM at $P_{\text {th }}>P_{\max }$ or CNM at $P_{\text {th }} \ll P_{\text {min }}$ then dynamical processes are likely to dominate with dynamical times much less than the cooling times. Experiments of this kind could be carried out with STO, SOFIA, and Herschel observations of Galactic and extragalactic sources.

The question of phases is not without controversy. A great review article by VázquezSemadeni (2009) is entitled "Are there phases in the ISM". Much of the controversy centers on Heiles \& Troland (2003) which has turned into a bit of an urban legend. The legend is that $50 \%$ of the gas mass is in thermally unstable temperatures and TI plays little role in creating CNM and WNM gas. It appears that most everyone misses that there are two distributions plotted in their Fig. 2. The distribution in temperatures for the in-plane gas shows $\sim 75 \%$ of the warm gas within the $7000-9000 \mathrm{~K}$ range exactly as expected for TI. Only $\% 25$ of the gas is outside this range, and when the CNM is included only $\sim 15 \%$ of the gas mass is at thermally unstable temperatures. The out of plane distribution looks nothing like the in-plane distribution with much of the gas outside of 7000-9000 K. I would conclude that the in-plane gas is dominated by TI, while the out of plane is dominated by dynamical processes. Numerical simulations give mixed results showing either no or weak TI (e.g., Gazol et al. (2001)) or significant TI (e.g., Koyama \& Ostriker (2009)). The results depend on the model resolution, heating rates, cooling rates, and type and amplitude of the turbulence (Gazol et al. 2005).

\section{Acknowledgements}

M. G. W. was supported in part by a NASA LTSA grant \#NNG05G64G.

\section{References}

Gazol, A., Vázquez-Semadeni, E., \& Kim, J. 2005, ApJ, 630, 911

Gazol, A., Vázquez-Semadeni, E., Sánchez-Salcedo, F. J., \& Scalo, J. 2001, ApJL, 557, L121

Heiles, C. \& Troland, T. H. 2003, ApJ, 586, 1067

Koyama, H. \& Ostriker, E. C. 2009, ApJ, 693, 1316

Kulkarni, S. R. \& Heiles, C. 1987, Interstellar Processes, 134, 87

Vázquez-Semadeni, E. 2009, in: M. de Avillez (ed.), The Role of Disk-Halo Interaction in Galaxy Evolution, EAS Publication Series (Paris: EDP), 2009 in press

Wolfire, M. G., McKee, C. F., Hollenbach, D., \& Tielens, A. G. G. M. 2003, ApJ 587, 278 